\title{
Mobility-Related Outcomes of Aquatic Training Programs in Children with Cerebral Palsy: A Randomized Trial
}

\author{
Yeshayahu Hutzler*1,2 and Miriam Getz ${ }^{3}$ \\ ${ }^{1}$ Academic College at Wingate, Israel \\ ${ }^{2}$ Israel Sport Center for the Disabled Ramat Gan, Israel \\ ${ }^{3}$ Kibbutzim College of Education, Technology and Dance, Tel Aviv, Israel
}

Received: 制: December 07, 2018; Published: 阱: December 19, 2018

*Corresponding author: Yeshayahu Hutzler, Academic College at Wingate, Israel

Abstract

Objective: To compare the outcomes of an aquatic interval training program (AITP) and a Halliwick training program (HTP) in young children with cerebral palsy (CP).

Methods: Seventeen children aged 3-6 years participated after randomized allocation to either an AITP or HTP. Land-based functional tests: Pediatric Berg Balance Scale (BBS), the 10-meter test, Timed Up and Go (TUG), and the Energy Expenditure Index (EEI), as well as an aquatic skill test (WOTA), were assessed by blinded assessors prior to and after interventions lasting four months (two weekly 30-min sessions).

Results: Significant improvement with a large effect size (ES; Cohen's $d>0.8$ ) was observed in the EEI only in the AITP group. TUG performance time and WOTA scores significantly improved, with a large ES in both training groups.

Conclusion: AITP appears to be more effective for increasing efficiency while walking. Participants in both training protocols similarly improved in balance and aquatic skill.

Keywords: Balance; Training; Gross Motor Function Classification System (GMFCS); Walking

Abbreviations: AITP: Aquatic Interval Training Program ; HTP: Halliwick Training Program ; CP: Cerebral Palsy; BBS: Berg Balance Scale ; TUG: Timed Up and Go ; EEI: Energy Expenditure Index; GMFM: Gross Motor Function Measure

\section{Introduction}

Cerebral palsy (CP) is the most common physical disability in childhood (2.6-2.9/1000 children) [1], and second to intellectual disability in terms of lifetime costs, with an average cost per person approaching a million dollars in 2003 [2], mostly during childhood and adolescence. The ability to walk is most often delayed and restricted, or even unattained, thus causing a primary concern to parents and therapists of children with CP [3-4]. A survey across Europe suggested that at five years of age $30 \%$ of children with $\mathrm{CP}$ still don't walk, and unaided walking was accomplished by only $54 \%$ at age five [5]. A similar trend has been reported in the USA whereby $58.1 \%$ of children with $\mathrm{CP}$ are able to walk independently at eight years [6]. Children with CP appear to have decreased levels of physical fitness that result in a decrease of functional abilities [7] and poor walking economy, which can lead to an early onset of fatigue [8]. Consequently, several therapeutic programs for children with CP have, since the late 1990s, increasingly incorporated exercise training components such as muscle strength $[7,9]$ and cardiovascular endurance [10-12]. A combination of these training modalities has been specifically recommended for children with CP [13].

Interval or circuit training is a modality often preferred in exercise training, due to its benefits to muscle and connective tissue as well as to the participant's cardiovascular system during the recovery phases [14]. This training modality has been developed from body building programs and usually includes a series of cycles in identified exercise activities with a scaling of the load and volume of repetitions in each cycle, depending on the type of energy source targeted [15]. This exercise modality has gained popularity in the general exercise literature [16], as well as with individuals with cerebral palsy [11]. For example, Unnithan and colleagues 
showed reductions in the energy cost of arm cranking and an improvement in peak $\mathrm{VO}_{2}$ with a combined strength and aerobicinterval training program, in children with CP [13]. Blundel and associates conducted a non-randomized ABA time series design in a group of eight children with CP aged 4-8 using circuit strength training, and reported significant and substantial (up to 150\%) functional increases [17]. Vershuren and colleagues reported significant differences in physical fitness components, including muscle strength and aerobic and anaerobic capacity in children and adolescents with CP who participated in an eight-month circuit training program [11].

They also reported gains in participation levels and quality of life. More recently, Aviram and associates compared the effectiveness of a continuous treadmill training program and a group circuit interval training program in a multi-center, matched pairs study with 95 mostly ambulatory adolescents with spastic $\mathrm{CP}$, and reported that while both groups improved, more progress and less diminished training effects on follow-up were documented in the circuit training group [18]. In addition, or as an alternative to exercise programs on land, the aquatic environment has also been utilized for mobility-enhancing activities in persons with disability at very young ages [19], and exhibits one of the most popular modalities of complementary medicine specifically in children with CP [20]. It is commonly perceived that exercising in the aquatic environment can improve muscle strength and endurance in children with CP [21,22]. The unique benefits of the aquatic environment have been reported elsewhere [21-23]. Yet, the specific mechanisms of the aquatic environment that might contribute to the development of functional capacity in participants with neuromotor disorders have not yet been specified. However, some authors have compared running in the water to land or treadmill running. For example, Svedenhag and Seger indicated several differences between running on land and in deep water [24]. They showed that heart rates (HR) were lower for a similar $\mathrm{VO}_{2}$, while the blood lactate levels and perceived exertion were higher, after training in the water. Several phenomena appear to contribute to these differences:

a) The increased resistance to movement initiation and continuation in the water, caused by the water viscosity, which may add to the anaerobic component of muscle activation during an aquatic compared to a similar land-based activity [25-26],

b) This increased water resistance, together with the buoyancy effect of water, suppressing antigravity muscular work, which appear to reduce muscle blood flow and decrease the arterio-venous oxygen difference [24].

c) Movement patterns evoked during running in water that included a greater proportion of arm work compared to similar exercise on land [24].

However, only a few studies have provided evidence for beneficial cardiorespiratory effects of aquatic exercise in children with CP. Hutzler and colleagues reported significant increase in respiratory function in 23 children with $\mathrm{CP}$ who participated in a six-month combined aquatic and land-based program, compared to children who participated in a land-only exercise program [27]. In an additional study, Getz et al. reported significant differences in the metabolic cost of walking in kindergarten children with $\mathrm{CP}$ who participated in a four-month aquatic exercise program, in comparison to children who participated in a land-based exercise program [28]. Fragala-Pinkham and associates reported significant differences in walking endurance as measured by the half-mile walk/run in 16 children with various disabilities who participated in a 14-week aquatic aerobic group exercise program [29]. Similar outcomes were reported in a study of a group of eight ambulatory children and adolescents with CP (age range 6-15) [30]. In a larger sample of 19 children with CP, aged 6-12 years, who participated in a 12-week aquatic exercise program twice a week, Dimitrijević and associates reported significant increases in cardiorespiratory function as measured in maximal oxygen consumption [31].

In an additional study, Ballaz and colleagues reported a significant reduction of the Energy Expenditure Index (EEI) and working HR while walking, in a group of 10 ambulatory adolescents with $\mathrm{CP}$, following 20 aquatic treatment sessions [32]. It should be noted that the majority of studies reporting on pediatric aquatic interventions have used prospective, one-group designs, which are considered to have a low evidence level. Furthermore, most of the aquatic intervention programs reported were based on the traditional "Halliwick" concept, developed in the late 1940s and early 1950's by James McMillan, with the intention of teaching independence in the aquatic environment, teaching swimming skills, and promoting participation in aquatic activities for persons with disability and particularly CP $[28,33]$. The Halliwick training program (HTP) follows 10 points, focusing on respiratory adjustment, hydrodynamic orientation, longitudinal rotations, sagittal rotations, and the aquatic progression method [33-35]. Due to the focus on aquatic skilled mobility as an outcome of the ability to control rotations in in the three movement dimensions, HTP can be described as a non-specific training modality of mobility, with low to moderate intensity. In contrast, Fragala-Pinkham and associates reported on a moderate intensity interval training-based program, including swimming laps, relay competitions, and aquatic games [29]. However, this study was performed with a mixed group that included participants with various disabilities, and there was no comparison group. Furthermore, based on a recent systematic literature review, it was concluded that aquatic exercise is feasible with minimal adverse effects, but that the dosing parameters are unclear [36].

Given the reported evidence of interval training in children with $\mathrm{CP}$ on one hand and the specific effects of training these children in aquatic environment on the other hand, the purpose of this study was to explore and compare the functional mobility outcomes on land of a purposefully designed aquatic interval training program (AITP) focusing on repetitive bouts of assisted kicking and swimming training compared to a traditional training program based on HTP. A secondary aim was to explore the associations between outcomes. The specific research aims were: 
a) To assess the effectiveness of the AITP compared to the HTP on functional mobility outcomes on land in children with $\mathrm{CP}$

b) To assess the effectiveness of AITP compared to HTP on aquatic skill outcomes in children with CP.

c) To assess the relationship between the functional mobility outcomes on land and the aquatic skill outcomes in children with CP.

d) To assess the relationship between the changes in functional mobility outcomes on land after the intervention and the participants' entry mobility level before the intervention.

\section{Method}

\section{Design}

The study was designed as a double blinded, (participants and assessors) randomized groups, controlled clinical trial. Pretests were conducted following a summer break and a two-week habituation period to the aquatic environment following the summer vacation. The habituation period was designed to allow all participants, to adjust (for new participants) or readjust (for those who continued from last year's activity) to the aquatic program environment, including the pool, personnel and aquatic routine. The aquatic intervention in both the AITP and HTP modalities were performed twice a week for a period of about four months. Each session lasted 30 minutes in an indoor heated therapeutic swimming pool (water temperature set at $33-34^{\circ} \mathrm{C}$ ). Post-test measurements were conducted after the intervention period. Children who missed more than $25 \%$ of the sessions due to illness were given additional sessions, up to completing attendance in 24 sessions. The study procedure was approved by the institutional review board of the Academic College at Wingate, as well as the Chief Scientist of the Ministry of Education and the Helsinki Committee of Hospital Asaf Harofeh Medical Center.

\section{Participants}

Fifty children with cerebral palsy, placed in two special education kindergarten centers that included aquatic intervention in their educational program, comprised the original study group. Participants had to meet the following inclusion criteria:

\section{a) Medical diagnosis of $\mathrm{CP}$}

b) No other medical complications such as seizures during the previous year

\section{c) Compliance and ability to comprehend instructions}

d) Did not have any medical procedures involving the lower limbs in the last 12 months (including casting or Botulinum injections)

e) Being able to walk independently and continuously for three minutes

f) Children's parents or guardians signed an informed consent form.

The participants' enrollment, allocation, and analysis are shown in (Figure 1). Randomization was completed by a research assistant who was not familiar with the participants, did not take part in the intervention and was independent of the study. Randomization was performed by taking into consideration pairs of children at similar age and function ranges by means of stratification. Function was measured by means of the Gross Motor Function Classification System (GMFCS) [37]. This system differentiates between five classification levels (levels 1 and 2 - children are independently mobile; level 3 - children typically are using mobility aids; and levels 4 and 5 - children mostly use wheelchair mobility). After randomization of the 22 children who acquired parental informed consent into two groups of 11 participants each, two children (one in each group) were excluded during pre-test measuring due to their difficulty in completing the pre-test testing protocol. As to the posttest measurement, three additional children from the HTP and one child from the AITP were excluded due to leaving the kindergarten or to medical conditions. Thus, the final sample included nine children in the AITP and seven children in the HTP. In addition to GMFCS we also acknowledged participants' clinical CP description (hemiplegia, diplegia, tetraplegia), but this was not taken into account for randomization.

\section{Outcome Measures}

Four functional mobility field tests were used for measuring balance (pediatric balance scale), agility (timed up \& go), endurance (Energy Expenditure Index) and walking velocity (10m walk) as described below:

\section{Energy Expenditure Index (EEI)}

Predicted walking energy cost was calculated by extracting the resting HR from the steady state walking HR divided by the walking velocity, providing a score of heart beats per meter [38]. This score has been used in participants with CP, for assessing endurance capacity $[13,28]$. The EEI protocol included:

a) The participant was equipped with a polar HR monitor

b) The participant was asked to sit quietly for a period of 5 minutes, recorded HR of the last 2 minutes were averaged to determine resting $\mathrm{HR}$ values

c) The participant was asked to walk at a self-paced speed, (with all walking accessories usually used during walking) for a period of 4 minutes to ensure reaching steady state values

d) After 4 minutes the child continued walking for another minute, averaged HR, distance walked (in meters) and number of steps were recorded.

\section{Timed Up and Go Test (TUG)}

The TUG test [39] measures the total time spent for rising from a chair, locomotion for three meters, turning around an object, and returning to sit on the chair. It was originally developed for older persons, but has been clinically tested in children and adolescents with $\mathrm{CP}$, and found to have good correlation with the Gross Motor Function Measure (GMFM) [40]. We decided to employ the adult version [39] because several of the participants showed difficulties in completing the children version of the TUG [41]. Specifically, participants with walkers showed major difficulties turning around after touching the star on the wall and returning to the stool. 


\section{0-m Test (10mT)}

This test is utilized for measuring velocity of walking a short distance under two conditions:

a) Walking as fast as possible without running

b) Walking at regular speed.

An observer uses a stopwatch to measure the time it takes the child to walk the 10 meter distance, from the time the foot touches the starting line and until the foot contacts the end of the 10 meter line. Participants perform three trials under each condition and rest between trials until the HR is within $10 \%$ of resting values. Velocity ( $\left.\mathrm{m}^{*} \mathrm{sec}-1\right)$ is calculated by dividing the averaged time scores under each condition by the distance $(10 \mathrm{~m})$. The $10 \mathrm{mT}$ was found valid and is very popular for measuring mobility in children with CP [42].

\section{Pediatric Balance Scale (PBS)}

The PBS is a measure of balance, consisting of 14 tasks that challenge static as well as dynamic balance, while the participant is sitting, standing, or taking steps. This measure was originally developed for the elderly, [43] but was found valid and reliable in participants with CP [44]. The items are scored on a four-point scale $(0=$ unable to perform to $3=$ performs at a normative age-related manner). Thus, the total score range is 0 to 56 , where higher scores indicate better balance performances.

An additional instrument was used to measure aquatic skill performance new Section.

\section{Water Orientation Test Alyn (WOTA)}

This is a test developed for assessing the degree of water orientation and swimming readiness in children with a developmental disability, and is based on the principles of the HTP. It includes 27 items on a scale between 0 (can't perform) and 3 (fully performs), with a total score rage between 0 and 81 . The test proved to have good reliability (ICC reliability $=.97$ ) and moderate convergent validity ( $r=.57-60)$ compared to another test, the Brief Assessment of Motor Function [45].

\section{Intervention Protocols}

After randomization into the two training groups, each child was assigned to an individual instructor who was instructed to provide the specific intervention assigned to the child. All instructors were certified in the HTP and had a total training volume of 350 to 500 hours in hydrotherapy. In addition, all instructors received a sixsession course in the specific theory and practice of the AITP. The AITP protocol was based on an interval circuit training routine. Each exercise was designed to include intensive repetitive practice of an exercise designed for the aquatic environment. Each exercise included five sets of 4-6 repetitions across a 15 meter distance. The exercises included: progression in a prone position propelling arms or legs; diving in a prone position; progression in a supine position while propelling arms or legs; and, propelling legs in upright position. Fifteen-second intervals were given every time the child discontinued the exercise. The number of stops and HR were recorded throughout the intervention. Target HR was set at $60 \%$ max across participants including a $10 \%$ HR reduction due to the aquatic properties.

Instructors support was given when needed in order to maintain target HR throughout the session. The HTP protocol was based on the traditional approach, and in addition to warmup and cool-down activities performed in a group it included five individual activities of transferring between body positions, from the following repertoire: entering the pool through a prone glide; a supine glide from the instructor to the wall and back; progressing along the pool wall with the hands for a two-meter distance, transferring from water to a sitting position on a mattress and back to the pool; and, transferring from a supine floating position to an upright position.

\section{Statistical Analyses}

The small sample size did not permit an analysis of covariance [46] Normal distribution of the scores was assessed by means of the D'Agostino-Pearson measure [47]. All scores except for the TUG pre- and post-test were normally distributed. Therefore, while the other measures were tested by means of t-tests, the TUG results were analyzed using A-parametric tests (Wilcoxon for between groups and Mann-Whitney U-tests for within-group analyses. In addition, Cohen's d effect sizes (ES) [48] were measured with the Morris and Deshon's correction [49]. ES below .50 were considered trivial, between .51 - .80 moderate, and above 80 large.

In order to comply with the third research aim, Pearson's $r$ (or Spearman's R in variables associated with TUG) correlations were computed between the functional mobility and aquatic skill tests. Similarly, correlations were computed between the change scores gained during the intervention and the pre-test scores of the same tests, addressing the fourth research aim.

\section{Results}

A description of demographic variables of the final sample across both intervention groups are presented in (Table 1). As can be seen in (Table 1), the final sample is similarly distributed across age and GMFCS classes III-IV, but for GMFCS classes I and II there were five AITP participants, compared to only three in the HTP. This disproportion occurred due to the discontinuation of more individuals from the HTP (Figure 1). However, it is difficult to foresee how this may have affected the results. In order to respond to research aims one and two regarding the impact of intervention type, analyses were performed between groups across the mobility and aquatic skill variables. Descriptive data of pre- and post-test assessments, as well as results of the statistic tests, are presented in (Table 2). The ES in each of the intervention programs can be seen in (Figure 2). The data indicate that no significant effects were obtained in either intervention for the Berg and 10-m tests. However, a significant effect with a fairly similar size was found in the TUG test measuring dynamic balance and the WOTA measuring aquatic skills. The ES were large in the WOTA, and moderate in the TUG. 


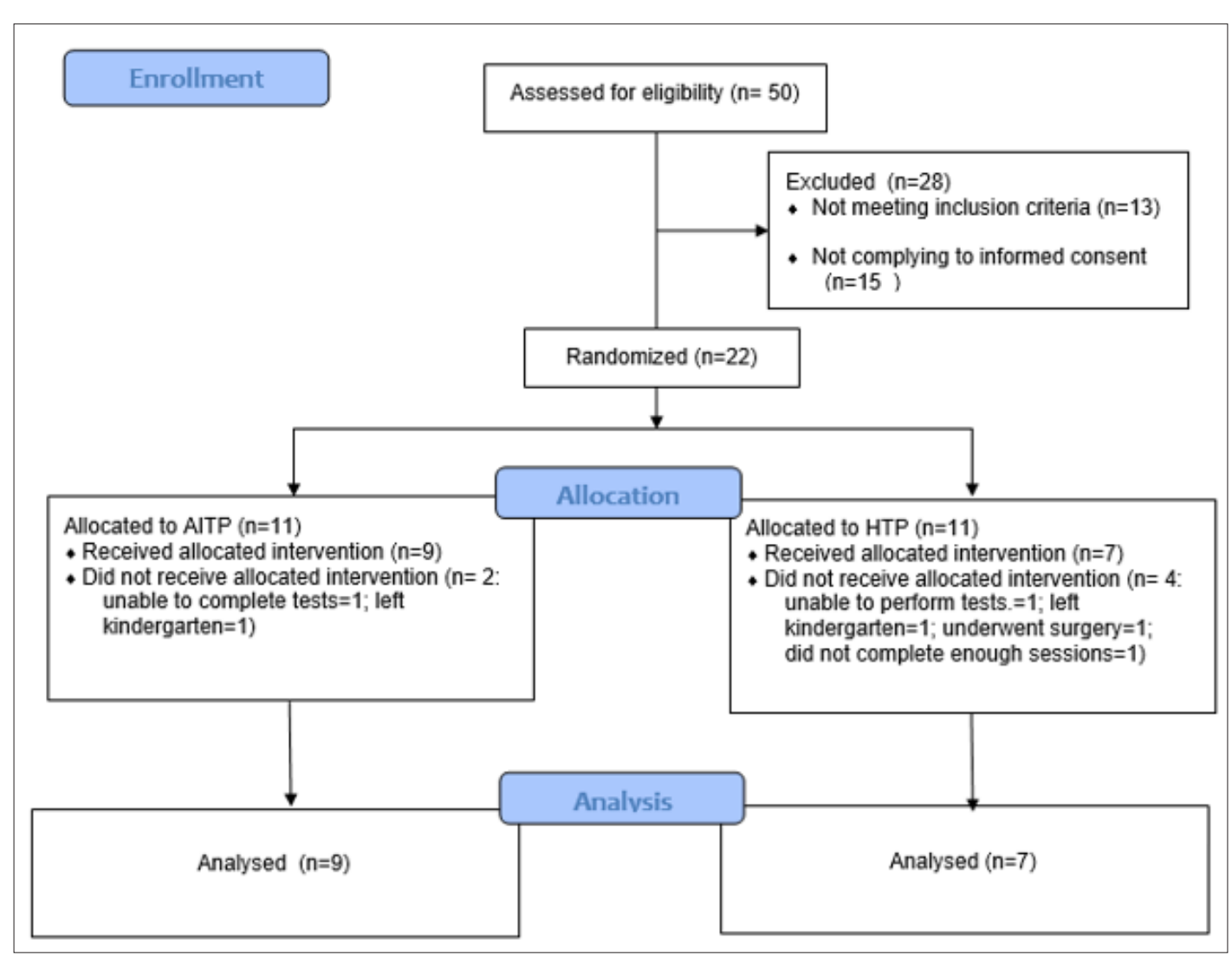

Figure 1: CONSORT 2010 flow chart presenting participant enrollment, allocation and analysis.

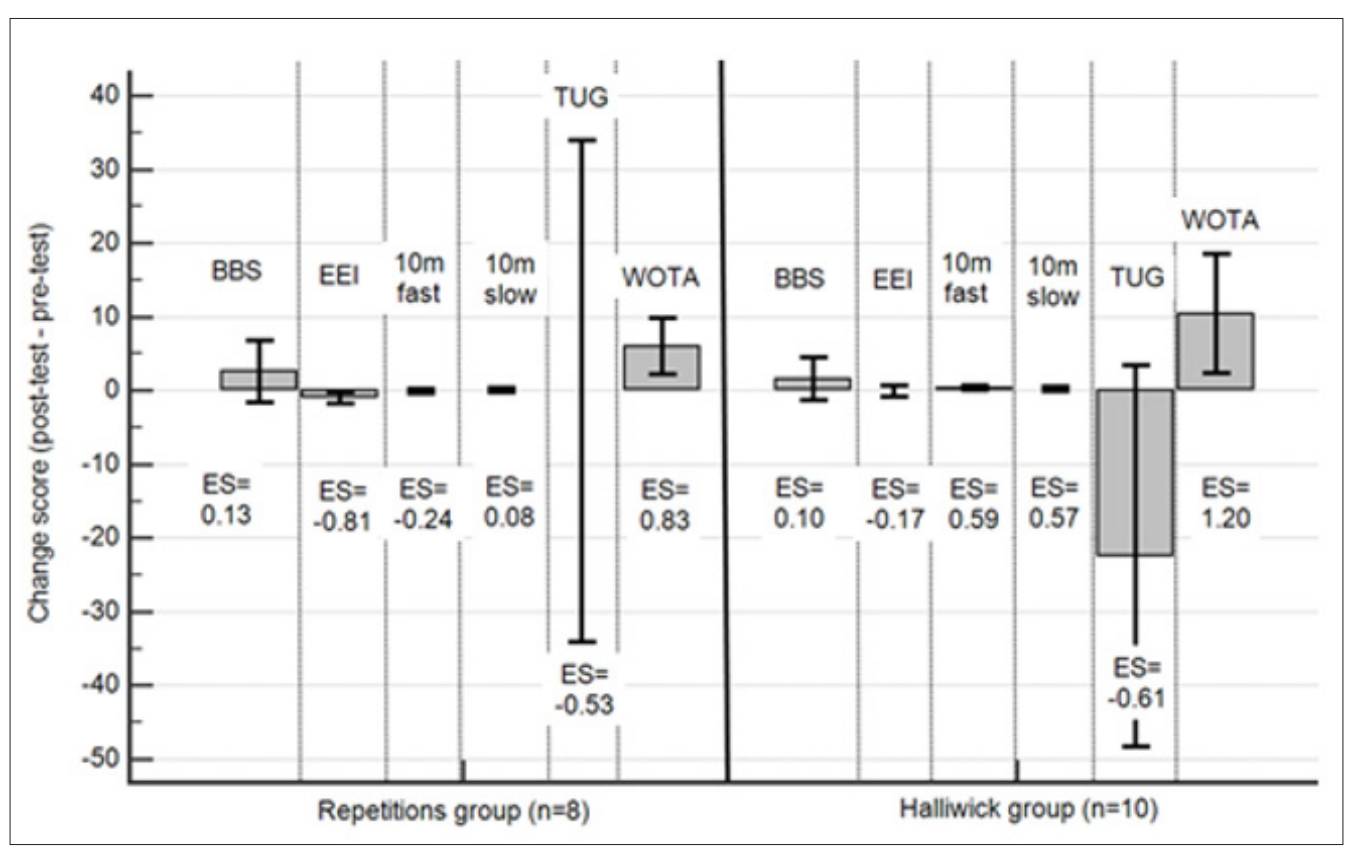

Figure 2: Change scores and effect sizes - by treatment group.

BBS, Berg Balance Scale; EEI, energy expenditure index; TUG, Timed Up and Go Test; 10m fast, 10 meter walk test - fast gait; $10 \mathrm{~m}$ slow, ten meter walk test - comfortable gait; Cohen's d calculation: mean $\Delta$ /standard deviation average from two means (large differences: Cohen's d > 0.80, moderate differences: 0.51-0.80; Cohen's d is based on a single pooled standard deviation; Cohen's d was corrected for dependence between means, using Morris and DeShon's47 equation; The bars represent change scores mean; The error bars represent $95 \%$ confidence interval for mean. 
Table 1: Distribution of participants according to gender, age, and level of activity across groups.

\begin{tabular}{|c|c|c|}
\hline Characteristics & AITP & HTP \\
\hline & Age (y:m) & \\
\hline Mean (SD) & 5:95 (1.18) & $5: 35(1.32)$ \\
\hline \multicolumn{3}{|c|}{ Gender } \\
\hline Male & 6 & 5 \\
\hline Female & 3 & 2 \\
\hline \multicolumn{3}{|c|}{ GMFCS } \\
\hline I & 1 & 3 \\
\hline II & 4 & \\
\hline III & 4 & 3 \\
\hline IV & & 1 \\
\hline \multicolumn{3}{|c|}{ Type of Cerebral Palsy } \\
\hline Hemiplegia & 4 & 3 \\
\hline Diplegia & 4 & 3 \\
\hline Quadriplegia & & 1 \\
\hline Ataxia & 1 & \\
\hline
\end{tabular}

Note: AITP, Aquatic interval training program; HTP, Halliwick training program; GMFCS, Gross Motor Classification System Table 2: Outcome variables of participants.

\begin{tabular}{|c|c|c|c|c|c|c|c|c|c|}
\hline \multirow{2}{*}{ Variables } & \multicolumn{3}{|c|}{ Entire Group ( $\mathrm{N}=18)$} & \multicolumn{3}{|c|}{ AITP Group (N= 9) } & \multicolumn{3}{|c|}{ HTP Group (N=7) } \\
\hline & Pre-Test & Post-Test & $\begin{array}{l}\text { Change } \\
\text { Score }\end{array}$ & Pre-Test & Post-Test & $\begin{array}{l}\text { Change } \\
\text { Score }\end{array}$ & Pre-Test & Post-Test & $\begin{array}{c}\text { Change } \\
\text { Score }\end{array}$ \\
\hline $\begin{array}{c}\text { Berg Balance Scale, } \\
\text { mean (SD) }\end{array}$ & $\begin{array}{c}30.00 \\
(16.97)\end{array}$ & $\begin{array}{c}32.00 \\
(17.71)\end{array}$ & $2.00(4.00)$ & $\begin{array}{c}25.42 \\
(19.92)\end{array}$ & $\begin{array}{c}28.00 \\
(21.06)\end{array}$ & $2.57(4.50)$ & $\begin{array}{c}33.55 \\
(14.48)\end{array}$ & $\begin{array}{c}35.11 \\
(15.19)\end{array}$ & $\begin{array}{c}1.55 \\
(3.77)\end{array}$ \\
\hline $\begin{array}{l}\text { EEI (beats/meter), } \\
\text { mean (SD) }\end{array}$ & $\begin{array}{c}1.82 \\
(1.23)\end{array}$ & $\begin{array}{c}1.28 \\
(0.90)^{*}\end{array}$ & $\begin{array}{l}-0.53 \\
(1.05)\end{array}$ & $\begin{array}{c}2.00 \\
(1.49)\end{array}$ & $\begin{array}{c}1.00 \\
(0.89)^{*}\end{array}$ & $\begin{array}{l}-0.99 \\
(0.93)\end{array}$ & $1.67(1.04)$ & $1.51(0.89)$ & $\begin{array}{l}-0.16 \\
(1.02)\end{array}$ \\
\hline $\begin{array}{c}\text { Ten meters - fast } \\
\text { (meters/ second), } \\
\text { mean (SD) }\end{array}$ & $\begin{array}{c}0.85 \\
(0.54)\end{array}$ & $\begin{array}{c}0.98 \\
(0.56)\end{array}$ & $0.12(0.44)$ & $\begin{array}{c}0.79 \\
(0.60)\end{array}$ & $\begin{array}{c}0.67 \\
(0.44)\end{array}$ & $\begin{array}{l}-0.12 \\
(0.31)\end{array}$ & $0.90(0.52)$ & $\begin{array}{c}1.22 \\
(0.54) \mathrm{a}\end{array}$ & $\begin{array}{c}0.31 \\
(0.45)\end{array}$ \\
\hline $\begin{array}{c}\text { Ten meters- slow } \\
\text { (meters/second), } \\
\text { mean (SD) }\end{array}$ & $\begin{array}{c}0.64 \\
(0.35)\end{array}$ & $\begin{array}{c}0.76 \\
(0.40)\end{array}$ & $0.12(0.30)$ & $\begin{array}{c}0.63 \\
(0.43)\end{array}$ & $\begin{array}{c}0.67 \\
(0.44)\end{array}$ & $0.03(0.27)$ & $0.64(0.30)$ & $0.84(0.38)$ & $\begin{array}{c}0.19 \\
(0.31)\end{array}$ \\
\hline $\begin{array}{c}\text { TUG (seconds), } \\
\text { Median (95\% CI) }\end{array}$ & $\begin{array}{c}22.55 \\
(13.04- \\
44.79)\end{array}$ & $\begin{array}{c}14.21 \\
(8.43- \\
29.08)^{*}\end{array}$ & $\begin{array}{l}-12.70 \\
(8.43- \\
44.79)\end{array}$ & $\begin{array}{c}22.55 \\
(13.04- \\
44.79)\end{array}$ & $\begin{array}{c}14.21 \\
(8.43- \\
29.08)^{*}\end{array}$ & $\begin{array}{l}-0.13 \\
(8.43- \\
44.79)\end{array}$ & $\begin{array}{c}26.26 \\
(15.71- \\
59.98)\end{array}$ & $\begin{array}{c}11.40 \\
(8.45- \\
30.96)^{*}\end{array}$ & $\begin{array}{l}22.48 \\
(8.45- \\
59.98)\end{array}$ \\
\hline WOTA, mean (SD) & $\begin{array}{l}33.31 \\
(6.81)\end{array}$ & $\begin{array}{c}41.73 \\
(8.70)^{*}\end{array}$ & $8.42(8.70)$ & $\begin{array}{l}34.75 \\
(7.88)\end{array}$ & $\begin{array}{c}40.75 \\
(6.40)^{*}\end{array}$ & $6.00(4.50)$ & $\begin{array}{l}31.60 \\
(5.98)\end{array}$ & $\begin{array}{c}42.0 \\
(10.74)^{*}\end{array}$ & $\begin{array}{c}10.40 \\
(11.23)\end{array}$ \\
\hline
\end{tabular}

Note: *,Significant within group differences ( $<<0.05)$; a, significant difference between AITP and HTP groups; ( $<$ < 0.05); CI, confidence interval; EEI, Energy Expenditure Index; SD, standard deviation; TUG, Timed Up and Go Test; According to D’ AgostinoPearson test, all variables but for TUG are normally distributed. In normally distributed variables paired and independent $\mathrm{t}$-tests were used. In non-normally distributed variables Wilcoxon and Mann-Whitney U-tests were used. AITP, Aquatic Interval Training Program; HTP, Halliwick Training program. 
As can be seen in (Table 2) differences between both intervention programs were observed. The participants in the AITP significantly improved their EEI that is they reduced their HR beats per meter walked, while the HTP did not change. In contrast, the participants in the HTP improved performance in the fast walking 10-m test, while the participants of the AITP slightly reduced their performance, leading to a significant difference between groups in the posttest. (Table 3) presents the correlations between the functional mobility and the aquatic skill variables, indicating a significant moderate association between the pre-test score in the $10-\mathrm{m}$ fast walking subtest and the mean aquatic skill (WOTA) score. (Table 4) presents the associations between the change score gained during gteh intervention and the pre-test scores. The associations in the EEI and TUG tests were moderate $(r=-0.695)$ and strong ( $\mathrm{r}=-0.894)$, respectively [50].

Table 3: Correlations between change scores in functional measures and WOTA.

\begin{tabular}{|c|c|c|c|c|c|c|c|}
\hline Measure & Group & $\begin{array}{l}\text { Berg Balance } \\
\text { Scale - } \\
\text { Change Score }\end{array}$ & $\begin{array}{c}\text { Ten Meter } \\
\text { Walk Test - } \\
\text { Fast - Change } \\
\text { Score }\end{array}$ & $\begin{array}{c}\text { Ten Meter } \\
\text { Walk Test } \\
\text { - Slow - } \\
\text { Change Score }\end{array}$ & $\begin{array}{l}\text { Timed Up } \\
\text { And Go } \\
\text { - Change } \\
\text { Score }\end{array}$ & $\begin{array}{l}\text { Energy Expendi- } \\
\text { ture Index -Change } \\
\text { Score }\end{array}$ & $\begin{array}{c}\text { WOTA - Change } \\
\text { Score }\end{array}$ \\
\hline \multirow{3}{*}{$\begin{array}{l}\text { Berg Balance } \\
\text { Scale -change } \\
\text { score }\end{array}$} & Entire group & ----- & -0.26 & -0.09 & 0.34 & -0.30 & 0.44 \\
\hline & AITP & $-\cdots-$ & -0.42 & -0.28 & 0.56 & -0.65 & 0.622 \\
\hline & HTP & ------ & -0.13 & 0.12 & 0.08 & 0.03 & 0.54 \\
\hline \multirow{3}{*}{$\begin{array}{l}\text { Ten meter walk } \\
\text { test - fast - } \\
\text { change score }\end{array}$} & Entire group & $-\cdots--$ & ----- & $0.83^{*}$ & -0.14 & 0.41 & -0.09 \\
\hline & AITP & $-\cdots$ & ----- & 0.67 & -0.04 & 0.50 & -0.23 \\
\hline & HTP & ----- & ------ & $0.91^{*}$ & 0.06 & 0.14 & -0.32 \\
\hline \multirow{3}{*}{$\begin{array}{l}\text { Ten meter walk } \\
\text { test - slow - } \\
\text { change score }\end{array}$} & Entire group & ----- & ----- & ----- & -0.00 & 0.47 & -0.23 \\
\hline & AITP group & ------ & ----- & ----- & 0.14 & 0.68 & -0.59 \\
\hline & HTP group & ------ & ------ & ----- & 0.06 & 0.23 & -0.30 \\
\hline \multirow{3}{*}{$\begin{array}{l}\text { Timed Up and Go- } \\
\text { change score }\end{array}$} & Entire group & $-\cdots---$ & ----- & ----- & ----- & -0.24 & 0.03 \\
\hline & AITP group & ----- & ----- & ----- & ----- & -0.42 & 0.37 \\
\hline & HTP group & ------ & ------ & ----- & ------ & -0.11 & 0.08 \\
\hline \multirow{3}{*}{$\begin{array}{l}\text { Energy expen- } \\
\text { diture index- } \\
\text { change score }\end{array}$} & Entire group & ----- & ----- & ----- & ----- & ----- & $-0.47^{*}$ \\
\hline & AITP group & --.-- & ----- & ----- & ----- & ----- & $-0.80^{*}$ \\
\hline & HTP group & ----- & ----- & ------ & ----- & ------ & $-0.64^{*}$ \\
\hline \multirow{3}{*}{$\begin{array}{l}\text { WOTA - change } \\
\text { score }\end{array}$} & Entire group & ----- & $-\cdots--$ & ----- & ----- & $-\cdots--$ & $-\cdots--$ \\
\hline & AITP group & ----- & $-\cdots--$ & ----- & $-\cdot---$ & $-\cdots--$ & $-\cdot---$ \\
\hline & HTP group & ----- & ----- & ----- & ----- & ----- & ----- \\
\hline
\end{tabular}

Note: *,Significant at p < 0.05 (2=tailed); AITP , Aquatic Interval Training Program; HTP , Halliwick Training program 
Table 4: Correlations between pre-score and change score - entire group.

\begin{tabular}{|c|c|}
\hline Variables & $\begin{array}{c}\text { Correlation Between Pre-Test and Change } \\
\text { Score }\end{array}$ \\
\hline Berg Balance Scale & 0.073 \\
\hline EEI (beats/meter) & $-0.695^{*}$ \\
\hline $\begin{array}{c}\text { Ten meters - fast (me- } \\
\text { ters/second) }\end{array}$ & -0.376 \\
\hline $\begin{array}{c}\text { Ten meters - slow (me- } \\
\text { ters/second) }\end{array}$ & -0.234 \\
\hline TUG (seconds) & $-0.894^{*}$ \\
\hline WOTA & -0.392 \\
\hline
\end{tabular}

Note: *,Correlations are significant at the 0.05 level; EEI, Energy Expenditure Index; SD, standard deviation; TUG, Timed Up and Go Test; According to D'Agostino-Pearson test, all variables but for TUG are normally distributed. In normally and non-normally distributed variables Pearson and Spearman rank correlations were used, respectively.

\section{Discussion}

In this study kindergarten children with $\mathrm{CP}$ were randomly assigned into two intervention programs - an interval program specifically designed for the aquatic environment (AITP) and a traditional HTP, which is the preferred aquatic treatment for children with CP in Israel and in some other countries [23,28,31,51]. The current research focused on functional mobility outcomes on land as well as aquatic skill outcomes, which are an important asset for swimming participation throughout the life span, and a feasible and practical activity for persons with mobility restrictions. The research questions of this study concerned the specific impact of each intervention program on functional mobility and aquatic skill, as well as the relation of the functional mobility and aquatic skill outcomes and the impact of baseline performance on change scores.

\section{Effect on Functional Mobility Outcomes}

This is the first study known to the authors addressing the differential impact of aquatic intervention programs on functional mobility. Based on the well-established effectiveness of land-based interval training, we hypothesized that using this modality (AITP) may be more suitable than the aquatic skill-based HTP. However, the results portrayed in Table 2 indicate such an advantage only with regard to walking endurance using the EEI measure. The AITP group significantly reduced their HR while walking with a large ES (d-0.81), while the HTP group did not significantly change and demonstrated only a trivial ES $(\mathrm{d}=0.17)$. An improvement in EEI was also reported in previous studies concerning aquatic treatment in children with CP $[28,31,32]$. However, the overall percentage of EEI change in the current study was $30 \%$, which is twice the change reported in the study of Ballaz et al. [32] (15\%). This larger change proportion in the current study was mostly due to a $100 \%$ change observed in the AITP (Table 2). In addition, previous studies have reported a significant increase in walking economy [28] and peak oxygen consumption while walking [31] following an aquatic intervention. The operational meaning of this finding is an ability to maintain a faster pace and achieve a longer distance, while walking. A similar finding was reported by Fragala-Pinkham and associates, who demonstrated a significant reduction in walking time over a distance of half a mile following an aquatic intervention based on interval training principles in a heterogenic group of children with developmental disabilities [29].

In contrast, children in the HTP group improved their performance in fast walking throughout a short distance $(10 \mathrm{~m})$, with a moderate effect size $(\mathrm{d}=0.59)$, compared to the children in the AITP group, whose performance slightly declined. Furthermore, while not significantly within each group different, the children in the HTP group exhibited a slightly larger ES (d-0.61) compared to the children in the AITP group $(\mathrm{d}=0.52)$. It may be suggested that the recurrent experience of rotations in the three planes enabled in the viscosity-rich aquatic environment, and common in HTP, improved proprioceptive sensory regulation in HTP participants. The AITP does not focus on rotational exercises, and this may explain the difference. An important question is to what extent does the aquatic training add to therapeutic outcomes obtained using land-based treatment? Based on previous comparative research, it has been suggested that the metabolic cost of walking may be better impacted using the aquatic intervention, while fast walking may better be trained on land [28].

The outcomes obtained in the current study may be considered for designing future aquatic interventions. Given that aquatic intervention is mostly secondary to land-based exercise for increasing functional mobility, it appears reasonable to focus the aquatic intervention on what works best in this environment, which appears to be the endurance function. Therefore, adding interval training principles using sets of short kicking, diving and swimming bouts as a part of the aquatic intervention sessions is recommended.

\section{Effect on Aquatic Skills}

Previous studies addressing aquatic interventions have reported significant improvement in aquatic skills measured via WOTA or a similar measure obtained via the Aquatic Independence Measure [52]. The results obtained in the current study revealed that any of the interventions similarly contributed to participants' aquatic performance. The effect size observed in WOTA was similarly large $(d>0.8)$ in both groups, and represented a $25 \%$ increase in skill performance, which is quite similar (26\%) to what was reported across a similar period of 12 weeks in Israel, [45] but smaller than the gain reported in a Serbian study [31]. The reason for the smaller gain in the current study may be due to the younger age of the children in our study $(4.95+1.18$ and $5.35+1.32$ yrs. in AITP and HTP, respectively), compared to the mean age reported in the Serbian study $(9.56+2.37 y$ yrs. for the total group), as well as to the shorter session duration in the current 
study (30min) compared to the Serbian study (55min). It should be noted that acquiring water skills at a young age is useful for facilitating participation in recreational aquatic activity with family and friends of an older age. Mastering such skills may enable a child with CP to represent a normative ability in a socially valued activity, and thereby acquire respectful recognition from classmates or adult supervisors, leading to increased self-esteem. Furthermore, participation in swimming competitions organized for children with disability may promote a desire to assess their competence compared to peers, and thereby develop competitive aspirations. This is important given the apparent inequality in the competitive capacity of children with CP compared to their able-bodied peers. Based on the findings in the current study, it may be suggested that the AITP and HTP similarly contribute to acquiring aquatic skills.

\section{The Association Between Functional Mobility on Land and Aquatic Skill}

Based on previous studies, [45-50] we expected that a significant and moderate association will be found between functional mobility on land and aquatic skill variables [46,51]. Our findings did not support this, and only revealed significant moderate associations between the total aquatic score at pre-test and the time for self-paced and fast walking at post-test $(r=.463$ and $r=.529$, respectively). This finding indicates that those with a better preliminary aquatic performance eventually performed better on fast walking at post-test, suggesting some relation between functional mobility on land and in water. However, there were small associations between all other variables. Previous studies included more variables of functional mobility in sitting and in prone and supine positions, which are more similar to that which are common in the aquatic environment, while the current study focused on vertical functional mobility. Together with the smaller sample size, this may be one of the reasons for the lower associations in the current study.

\section{The Association Between Baseline and Change Scores}

Based on the principle of diminishing returns, [16] one may expect that a negative association will be found between the baseline score and the change post-intervention in functional mobility and aquatic skill - that is, those who had lower preliminary performance will profit more from the aquatic interventions. The outcomes of this study contradicted this hypothesis with regard to functional mobility. Indeed, a moderate to large negative association was found in two indices: EEI and TUG ( $\mathrm{r}=-.695$ and -.894 respectively; see Table 4). However, it should be noticed that the lower scores in these indices (beats / meter) and time per course, respectively) designate a better performance. The negative association in this case indicates that those who had a better baseline performance achieved larger change scores during the intervention. It may be suggested that in order to benefit from the aquatic exercise, participants need to have a minimal baseline kicking and other mobility capacity, which might not be available in the more severe cases, particularly in participants in the GMFCS class IV. An opposite trend was observed with regard to aquatic skill, where the association between baseline and change scores was negative and slightly below moderate ( $r=-.392)$, meaning that there was a trend of those who had a lower aquatic skill score at baseline to increase their change score during intervention more than those who had a higher performance. This finding apparently follows the principle of diminishing returns.

Based on the findings in this study, it may be recommended to utilize aquatic intervention for reducing energy cost and increasing walking stability, mainly in participants of GMFCS classes I - III. Aquatic intervention should still be relevant for those with the lower functional capacity (i.e. GMFCS classes IV - V), mainly for increasing aquatic skill and perhaps land-based mobility activities not associated with walking, such as lifting the body from a mat, rolling, and crawling. Some studies have indicated an improvement in GMFM levels of children with CP following aquatic intervention [28,30-32,51]. However, these studies used the composite score, and a differential analysis of upright (i.e. standing and walking) compared to lower level (i.e. sitting, rolling, and crawling) stability and mobility function has not been performed. Future research with larger samples per GMFCS class may contribute to a better understanding of the differential contribution of aquatic intervention to children with CP.

\section{Limitations}

This study has several limitations. The first is the small number of participants, leading to reduced statistical power, which resulted from the relative strict inclusion criteria and the substantial number of parents and guardians who were not willing to sign the consent form. In future studies, a greater effort should be made to encourage parental interest in the results of the study. This may be accomplished by providing parents with individual reports and a personal consultation based on the study's outcomes. One factor that interfered with such a procedure being performed in the current study was the strict confidentiality constraints requested by the Ministry of Education Chief Scientist. Another limitation is the type of clinical tests used. Future studies should include the GMFM or an alternative functional performance assessment to enable a more specific description and analysis of upright mobility compared to other forms of functional mobility outcomes. An additional limitation was the absence of follow-up measurements to address the sustainability of the effects of the intervention. This part of the study was not practiced due to lack of parental compliance to abandon the aquatic activity for a certain (even short) period of time. Finally, the difference between the groups in the distribution of children across the GMFCS level, occurring due to sample drop-outs may have influenced results.

\section{Summary and Recommendations}

In view of the outcomes of this study, and due to the increasing popularity of using aquatic environments for mobility training of children with $\mathrm{CP}$, it is recommended to further explore this intervention method as part of the comprehensive developmental treatment approach in these children. We suggest focusing on the AITP, particularly in order to address endurance in upright mobility activity. The HTP may be useful in addressing other types of mobility function, such as rolling, lifting, and crawling, but this hypothesis needs scientific support. Both types of aquatic training 
programs appear to similarly benefit aquatic skill development, which plays an important role in encouraging children with CP to participate in physical activity.

\section{References}

1. Maenner MJ, Blumberg SJ, Kogan MD, Christensen D, Yeargin AM, et al. (2016) Prevalence of cerebral palsy and intellectual disability among children identified in two U.S. National Surveys, 2011-2013. Annals of Epidemiology 26(3): 222-226.

2. (2004) Centers for Disease Control (CDC) Economic costs associated with mental retardation, cerebral palsy, hearing loss, and vision impairment-United States 2003. Morbity and Mortality Weekly Report 53(3): 57-59

3. Shumway Cook A, Woollacott MH (2007) Motor control: Theory and practical applications. In Shumway Cook A, Woollacott MH (Eds.) (3 ${ }^{\text {rd }}$ edn.), Philadelphia, PA: Lippincot.

4. Hof AL (1996) Scaling gait data to body size. Gait \& Posture 4(3): 222 223.

5. Beckung E, Hagberg G, Uldall P, Cans C (2008) Probability of walking in children with cerebral palsy in Europe. Pediatrics 121(1): e187-e92.

6. (2012) Centers for Disease Control and Prevention (CDC). Prevalence of autism spectrum disorders-Autism and Developmental Disabilities Monitoring Network, 14 sites, United States, 2008. Morbity and Mortality Weekly Report Surveillance Summaries 61(SS03): 1-19.

7. Fowler EG, Kolobe THA, Damiano DL, Thorpe DE, Morgan DW, et al. (2007) Promotion of physical fitness and prevention of secondary conditions for children with cerebral palsy: Section on pediatric research summit proceedings. Physical Therapy 87(11): 1-15.

8. Maltais E, Bar Or O, Galea VG Pierrynowsky M (2000) Use of orthoses lowers the $\mathrm{O}(2)$ cost of walking in children with cerebral palsy. Medicine \& Science in Sports \& exercise 33(2): 469-473.

9. Dodd KJ, Taylor NF, Damiano DL (2002) A systematic review of the effectiveness of strength-training programs for people with cerebral palsy. Archives of Physical Medicine and Rehabilitation 83(8): 11571164.

10. Vershuren O, Ketelaar M, Gorter JW, Helders PJM, Gorton JW (2008) Exercise programs for children with cerebral palsy: a systematic review of literature. American Journal of Physical Medicine and Rehabilitation 87(5): 404-417.

11. Vershuren O, Ketelaar M, Gorter JW, Helders PJM, Uiterwaal CSPM, et al. (2008) Exercise training program in children and adolescents with cerebral palsy: A randomized controlled trial. Archives of Pediatrics and Adolescent Medicine 161(11): 1075-1081.

12. Rogers A, Lynne Furler B, Brinks S, Darrah J (2008) A systematic review of aerobic exercise for children with cerebral palsy: an AACPDM evidence report. Developmental Medicine and Child Neurology 50(11): 808-814.

13. Unnithan VB, Katsimanis G, Evangelinou C, Kosmas C, Kandrali I, et al. (2007) Effect of strength and aerobic training in children with cerebral palsy. Medicine \& Science in Sports \& Exercise 39(11): 1902-1909.

14. Daussin FN, Zoll J, Dufour SP, Ponsot E, Lonsdorfer Wolf E, et al. (2008) Effect of interval versus continuous training on cardiorespiratory and mitochondrial functions: relationship to aerobic performance improvements in sedentary subjects. American Journal of Physiology Regulatory, Integrative, Comparative Physiology 295(1): R264-272.

15. Tesch PA (1992) Training for bodybuilding. In Komi PV (Eds.) Strength and power in sport. Oxford: Blackwell Scientific Publications, pp. 370381.

16. Wilmore JH, Costill D (1994) Physiology of sport and exercise. Champaign, IL: Human Kinetics.
17. Blundell SW, Shepherd RB, Dean CM, Adams RD, Cahill BM (2003) Functional strength training in cerebral palsy: a pilot study of a group circuit training class for children aged 4-8 years. Clinical Rehabilitation 17(1): 48-57.

18. Aviram R, Harries N, Namourah I, Amro A, Bar Haim S (2017). Effects of a group circuit progressive resistance training program compared with a treadmill training program for adolescents with cerebral palsy. Developmental Neurorehabilitation 20(6): 347-354

19. McManus BM, Kotelchuk M (2007) The effect of aquatic therapy on functional mobility of infants and toddlers in early intervention. Pediatric Physical Therapy 19(4): 275-282.

20. Hurvitz EA, Leonard C, Ayyanger R, Nelson VS (2003) Complementary and alternative medicine use in families of children with cerebral palsy. Developmental Medicine and Child Neurology 45(6): 364-370.

21. Becker BE, Cole AJ (2004) Comprehensive aquatic therapy. In Becker BE Cole AJ ( $2^{\text {nd }}$ Edn.), Oxford: Butterworth Heinman Medical.

22. Kelly J, Darrah J (2005) Aquatic exercise for children with cerebral palsy. Developmental Medicine and Child Neurology 47(12): 838-842.

23. Getz M, Hutzler Y, Vermeer A (2006) Effects of aquatic interventions in children with neuromotor impairments: A systematic review of the literature. Clinical Rehabilitation 20(11): 927-936.

24. Svedenhag J, Seger J (1992) Running on land and in water: comparative exercise physiology. Medicine \& Science in Sports \& Exercise 24(10): 1155-1160.

25. Nakanishi Y, Kimura T, Yokoo Y (1999) Maximal physiological responses to deep water running. Applied Human Science 18(2): 31-35.

26. Holmer I, Stein EM, Saltin B, Ecklom B, Astrand PO (1974) Hemodynamic and respiratory responses compared in swimming and running. Journal of Applied Physiology 37(1): 49-54.

27. Hutzler Y, Chacham A, Bergman U, Szeinberg A (1998) Effects of movement and swimming program on vital capacity and water orientation skills of children with cerebral palsy. Developmental Medicine and Child Neurology 40(3): 176-181.

28. Getz M, Hutzler Y, Vermeer A, Yarom Y, Unnithan V (2012) The effect of aquatic and land-based training on the metabolic cost of walking and motor performance in children with cerebral palsy: A pilot study. International Scholarly Research Network Rehabilitation ID 657979, p: 8.

29. Fragala Pinkham MA, Haley SM, O Neil M (2008) Group aquatic aerobic exercise for children with disabilities. Developmental Medicine and Child Neurology 50(11): 822-827.

30. Fragala Pinkham MA, Smith HJ, Lombard KA, Barlow C, O Neil ME (2014) Aquatic aerobic exercise for children with cerebral palsy: a pilot intervention study. Physiotherapy Theory and Practice 30(2): 69-78.

31. Dimitrijević L, Bjelaković B, Lazović M, Stanković I, Čolović H, et al. (2012) Aquatic exercise in the treatment of children with cerebral palsy. 140(11-12): 746-750.

32. Ballaz L, Plamondon S, Lemay M (2011) Group aquatic training improves gait efficiency in adolescents with cerebral palsy. Disability and Rehabilitation 33(17-18): 1616-1624.

33. Lambeck J, Stanat F (2001) The Halliwick concept, part I. Journal of Aquatic Physical Therapy 8: 6-11.

34. Lambeck J, Stanat F (2001) The Halliwick concept, part II. Journal of Aquatic Physical Therapy 9: 6-11.

35. Maes JP, Gresswell A (2010) The Halliwick concept for clients with cerebral palsy or similar conditions. BABTT Newsletter, p. 62.

36. Roostaei M, Baharlouei H, Azadi H, Fragala Pinkham MA (2016) Effects of aquatic intervention on gross motor skills in children with cerebral 
palsy: A systematic review. Physical \& Occupational Therapy in Pediatrics 37(5): 496-515.

37. Palisano RJ, Hanna SE, Rosenbaum PL, Russell DJ, Walter SD, et al. (2000) Validation of a model of gross motor function for children with cerebral palsy. Physical Therapy 80(10): 974-983.

38. Rose J, Gamble JG, Burgos A, Medeiros J, Haskell W (1990) Energy expenditure index of walking for normal children and for children with Cerebral Palsy. Developmental Medicine and Child Neurology 32(4): 333-340.

39. Williams EN, Caroll SG, Reddihough DS, Phillips BA, Galea MP (2005) Investigation of the timed "up \& go" test in children. Developmental Medicine and Child Neurology 47(8): 518-524.

40. Nicollini-Panisson RDA, Donadio, MVF (2013) Timed Up and Go test in children and adolescents. Revista Paulista de Pediatria 31(3): 377-383.

41. Dhote, SN, Khatri, PA, Gavnir SS (2012) Reliability of "Modified timed up and go" test in children with cerebral palsy. Journal of Pediatric Neuroscience 7(23): 96-100.

42. Thompson P, Beath T, Bell J, Jacobson G, Phair T, et al. (2008) Test retest reliability of the 10 meter fast walk and the six minute walk test in ambulatory school aged children with cerebral palsy. Developmental Medicine and Child Neurology 50(5): 370-376.

43. Berg K, Wood Dauphinėe S, Williams JI, Gayton D (1989) Measuring balance in the elderly: preliminary development of an instrument. Physiotherapy Canada 41(6): 304-311.

44. Franjoine MR, Gunther JS, Taylor MJ (2003) Pediatric balance scale: A modified version of the berg balance scale for the school-age child with mild to moderate motor impairment Pediatric balance scale: A modified version of the berg balance scale for the school age child with mild to moderate motor impairment 15(2): 114-128.

45. Tirosh R, Kats Leurer M, Getz M (2008) Halliwick-based aquatic assessments: Reliability and validity. International Journal of Aquatic Research and Education 2(3): 224-236.

46. Faul F, Erdfelder E, Buchner A, Lang AG (2013) G*Power Version 3.1.7 [computer software]. Universität Kiel, Germany.

47. D’Agostino R, Pearson ES (1973) Tests for departure from normality. Empirical results for the distributions of b2 and $\sqrt{b} 1$. Biometrika $60(3)$ : 613-622.

48. Cohen J (1988) Statistical power analysis for the behavioral sciences. In Cohen J(Eds.) ( $2^{\text {nd }}$ edn.), Mahwah, NJ: Lawrence Erlbaum.

49. Morris SB, De Shon RP (2002) Combining effect size estimates in metaanalysis with repeated measures and independent-groups designs. Psychological Methods 7(1): 105-125.

50. Vaščáková T, Kudláček M, Barrett U (2015) Halliwick concept of swimming and its influence on motoric competencies of children with severe disabilities. European Journal of Adapted Physical Activity 8(2): 44-49.

51. Getz M, Hutzler Y, Vermeer A (2006) Relationship between aquatic independence and gross motor function in children with neuro-motor impairments. Adapted Physical Activity Quarterly 3(4): 339-355.

52. Lai CJ, Liu WY, Yang TF, Chen CL, Wu CY, et al. (2015) Pediatric aquatic therapy on motor function and enjoyment in children diagnosed with cerebral palsy of various motor severities. Journal of Child Neurology 30(2): 200-208.
ISSN: 2574-1241

DOI: $10.26717 / B J S T R .2018 .12 .002247$

Yeshayahu Hutzler. Biomed J Sci \& Tech Res

This work is licensed under Creative

Commons Attribution 4.0 License

Submission Link: https://biomedres.us/submit-manuscript.php

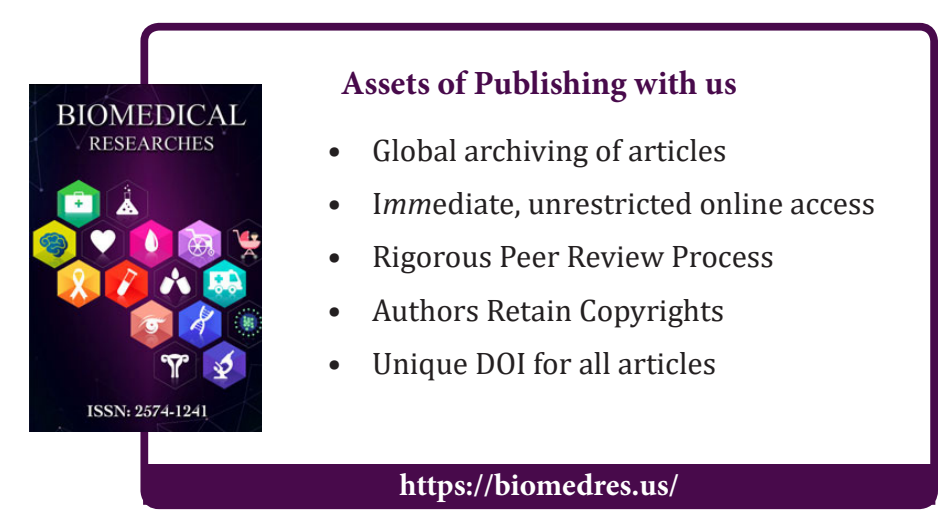

